\title{
Distribution center location selection using a novel multi criteria decision-making approach under interval neutrosophic complex sets
}

\author{
Mai Pham Quynha, Thuy Luong Thu ${ }^{a^{*}}$, Quynh Doan Huonga , Anh Pham Thi Vana, Hien Ngo Van ${ }^{b}$, \\ and Dan Nguyen Van ${ }^{a}$
}

${ }^{a}$ Academy of Finance, No. 58 Le Van Hien Street, Duc Thang Ward, Tu Liem District, Hanoi, Vietnam

${ }^{b}$ Phenikaa University, Yen Nghia Ward, Ha Dong District, Hanoi, Vietnam

\section{H R O N I C L E}

Article history:

Received November 28, 2019

Received in revised format:

December 28, 2019

Accepted January 24, 2020

Available online

February 1,2020

Keywords:

Distribution location selection

Multi-criteria decision-making

Neutrosophic set

Complex neutrosophic set

\begin{abstract}
A B S T R A C T
Distribution centers selection is a vital task of any company to reduce costs, improve efficiency of transport flows, which yields customer satisfaction. To select the suitable distribution centers, many quantitative and qualitative criteria must be considered in the selection process. Therefore, distribution centers selection can be seen as a multi-criteria decision making (MCDM) problem under vague environment. Single-valued complex neutrosophic sets (SVCNSs), which is generalized of fuzzy sets, complex fuzzy sets and intuitionistic fuzzy sets; can better represent the vague information than the other sets. This paper aims to propose a new the Technique for Order of Preference by Similarity to Ideal Solution (TOPSIS) approach based on SVCNSs to select the locations of distribution center. In the proposed TOPSIS approach, the importance weights of criteria, the ratings of alternatives, and their aggregated values are assessed and evaluated using SVCNSs. Then, this paper defines the operational rules of SVCNSs and calculates the aggregated weighted ratings of alternatives. Furthermore, the score, accuracy and certainty function are developed to rank the alternatives. Last, an application to the distribution center location selection is presented to show the advantages of the proposed approach.
\end{abstract}

\section{Introduction}

Distribution center location selection plays an important role for reducing the transportation cost, enforcing operation efficiency and logistic performance for distribution companies (Chen, 2001). In the process of selecting location for distribution centers, many objective and subjective criteria must be considered such as investment cost, climate condition, labor force quality and quantity, transportation availability, and closeness to demand market, etc. (Chen, 2001; Awasthi, et al., 2011; Chu \& Hsu, 2016; Li \& Wei, 2018). In addition, these criteria may have different importance (Chu \& Hsu, 2016; Greenfield et al., 2016). Therefore, the selection of distribution center location can be viewed as a multi-criteria decision making (MCDM) problem. Many precision-based methods for location selection have been proposed based on the concept of accurate measure and crisp evaluation. Intelligent heuristic algorithms are usually utilized to determine the optimal location of facilities (Rahmani \& Mirhassani, 2014; Hua et al., 2016; Hiassat et al., 2017; Toro et al., 2017; Zhalechian et al., 2017). Under many circumstances, the values for the locations are difficult to obtain with certainty due to the complexity and ambiguity of the decision environment. For such situations, the usage of 
precision-based methods is not adequate to solve the location selection problem (Li \& Wei, 2018). To solve with this problem, Zadeh (1965) proposed the fuzzy sets theory which is one of the most effective tools for processing vague information. Numerous fuzzy MCDM approaches have been proposed to solve the location selection problems. Awasthi et al. (2011) presented the technique for order of preference by similarity to ideal solution (TOPSIS) framework for location planning for urban distribution centers under a fuzzy environment. Rao et al. (2015) proposed a fuzzy multi-attribute group decision making (FMAGDM) technique based on a linguistic 2-tuple is used to evaluate potential city logistics centers locations from a sustainability perspective. Chu and Hsu (2016) applied a maximizing set and minimizing based fuzzy MCDM model to select and evaluate distribution center location. Pham et al. (2017) developed a fuzzy Delphi TOPSIS for choosing the locations of logistics centers in Vietnam. Singh et al. (2018) used a fuzzy analytic hierarchy process (AHP) for warehouse location selection. Recent studies in fuzzy sets have been extended by using complex fuzzy sets (Tamir et al., 2015; Selvachandran et al., 2018; Yazdanbakhsh \& Dick, 2018) for better designing and modeling reallife applications. The functionality of complex is for handling the information of uncertainty and periodicity simultaneously (Ali et al., 2018). Selvachandran et al. (2018) presented two new operations for the interval-valued complex fuzzy set model in order to solve an economics problem in the Malaysian economy in recent year. A systematic review of the field of complex fuzzy sets and logic was conducted by Yazdanbakhsh and Dick (2018) and Tamir et al. (2015). The application of fuzzy set theory allows incorporating the vague and imprecise linguistic terms into the decision process. However, the disadvantage of fuzzy sets is that it only has a membership, and is unable to express nonmembership. Atanassov $(1986,1989)$ introduced the intuitionistic fuzzy sets (IFSs) by adding a nonmembership function. IFSs can only handle incomplete information not the indeterminate information and inconsistent information. In order to process all kind of incomplete, indeterminate and inconsistent information, Smarandache $(1999,2005)$ proposed neutrosophic set (NS) by adding an independent indeterminacy-membership. Wang et al. (2005) further proposed a single valued neutrosophic set (SVNS) in order to directly use in real-life applications. SVNS is an instance of the NS. The SVNS, which is a generalization of fuzzy set and IFSs, has been magnificently applied in different fields, including decision-making problems (Pramanik et al., 2016; Das et al., 2019; Sodenkamp et al., 2018; Abdel-Basset et al., 2019; Abdel-Basset \& Mohamed, 2019; Liu \& You, 2019). However, a limited study has proposed the MCDM approaches using SVNSs for selecting and evaluating distribution center location (Pramanik et al., 2016). To our knowledge, no prior studies have developed a TOPSIS approach using the single-valued complex neutrosophic sets (SVCNS) to select and evaluate distribution center location.

This paper aims to propose a new TOPSIS approach based on SVCNS in order to support for distribution center location selection and evaluation process. In the proposed approach, the importance weights of decision criteria and the ratings of qualitative criteria are assessed in linguistic variables which are described by complex neutrosophic sets. This paper assesses and evaluates the importance weights of criteria, the ratings of alternatives, and their aggregated values using SVCNSs. Furthermore, the score, accuracy and certainty function are developed to rank the alternatives. Last, an application to the distribution center location selection is presented to show the advantages of the proposed approach. The organization of this paper is as follows. Section 2 introduces the concepts of complex fuzzy sets, NS, SVCNSs, and their operations. Section 3 presents the operational rules of the SVCNS. Section 4 proposed the TOPSIS approach in CNS. A numerical example with real dataset is used to show the procedure and advantage of the proposed method in Section 4. Finally, conclusion is given in Section 6.

\section{Preliminaries}

This section reviews the basic concepts of NS, complex fuzzy sets, and SVCN as in the following:

Definition 1. Neutrosophic Set (NS) (Smarandache, 1998)

Let $\mathrm{U}$ be a universe of discourse and a set $\mathrm{B} \subset \mathrm{U}$, such that 


$$
B=\left\{x,\left(T_{B}(x), I_{B}(x), F_{B}(x)\right), x \in U\right\},
$$

where $\mathrm{B}$ is characterized by a truth membership function $T_{B}$, an indeterminacy membership function $I_{B}$, and a falsehood membership function $F_{B} . \mathrm{T}_{\mathrm{B}}(\mathrm{x}), \mathrm{I}_{\mathrm{B}}(\mathrm{x}), \mathrm{F}_{\mathrm{B}}(\mathrm{x}) \subseteq[0,1]$ are real subsets, for all $\mathrm{x} \in$ $\mathrm{U}$, is called a neutrosophic set (NS) (Smarandache 1998). If $\mathrm{T}_{\mathrm{B}}(\mathrm{x}), \mathrm{I}_{\mathrm{B}}(\mathrm{x}), \mathrm{F}_{\mathrm{B}}(\mathrm{x}) \in[0,1]$ are real (crisp) numbers, for all $\mathrm{x} \in \mathrm{U}$, then $\mathrm{B}$ is called a single-valued neutrosophic set (SVNS) (Wang et al. 2005a).

\section{Definition 2. Complex Fuzzy Set}

Ramot et al. (2012) defined a complex fuzzy set A on a universe of discourse $\mathrm{U}$ as follows:

$A=\left\{\left(x, \lambda_{A}(x)\right): x \in U\right\}=\left\{\left(x, a_{A}(x) e^{j \omega_{A}(x)}\right): x \in U\right\}$,

where: $\lambda_{A}(x)=a_{A}(x) e^{j \omega_{A}(x)}, j=\sqrt{-1}, a_{A}(x) \in[0,1]$ and $\omega_{A}(x) \in[0,2 \pi]$ are both real value.

\section{Definition 3. Single-Valued Complex Neutrosophic Set}

A single-valued complex neutrosophic set (SVCNS) $\bar{R}$ on a universe of discourse $U$ can be represented in set form as (Ali and Smarandache, 2017):

$$
\bar{R}=\left\{\left(x, T_{\bar{R}}(x), I_{\bar{R}}(x), F_{\bar{R}}(x)\right): x \in U\right\}
$$

where: $T_{\bar{R}}(x)=o_{\bar{R}}(x) \cdot e^{j \mu_{\bar{R}}(x)}, I_{\bar{R}}(x)=u_{\bar{R}}(x) \cdot e^{j \lambda_{\bar{R}}(x)}$ and $F_{\bar{R}}(x)=v_{\bar{R}}(x) \cdot e^{j \omega_{\bar{R}}(x)}$ are the truth, indeterminacy and falsity membership function, respectively; $o_{\bar{R}}(x), u_{\bar{R}}(x), v_{\bar{R}}(x)$ and $\mu_{\bar{R}}(x), \lambda_{\bar{R}}(x), \omega_{\bar{R}}(x)$ are real values; $o_{\bar{R}}(x), u_{\bar{R}}(x), v_{\bar{R}}(x) \in[0,1]$ and $0 \leq o_{\bar{R}}(x), u_{\bar{R}}(x), v_{\bar{R}}(x) \leq 3$.

\section{Definition 4. Complement of Single-Valued Complex Neutrosophic Set}

The complement of a SVCNS $\bar{R}=\left\{\left(x, T_{\bar{R}}(x), I_{\bar{R}}(x), F_{\bar{R}}(x)\right): x \in U\right\}$ can be defined as:

$$
\bar{R}^{c}=\left\{\left(x, T_{\bar{R}^{c}}(x), I_{\bar{R}^{c}}(x), F_{\bar{R}^{c}}(x)\right): x \in U\right\},
$$

where,

$$
\begin{aligned}
& T_{\bar{R}^{c}}(x)=o_{\bar{R}^{c}}(x) \cdot e^{j \mu_{\bar{R}^{c}}(x)}=v_{\bar{R}}(x) \cdot e^{j\left(2 \pi-\mu_{\bar{R}}(x)\right)}, \\
& I_{\bar{R}^{c}}(x)=u_{\bar{R}^{c}}(x) \cdot e^{j \lambda_{\bar{R}^{c}}(x)}=\left(1-u_{\bar{R}}(x)\right) \cdot e^{j\left(2 \pi-\lambda_{\bar{R}}(x)\right)}, \\
& F_{\bar{R}^{c}}(x)=v_{\bar{R}^{c}}(x) \cdot e^{j \omega_{\bar{R}^{c}}(x)}=o_{\bar{R}}(x) \cdot e^{j\left(2 \pi-\omega_{\bar{R}}(x)\right)} .
\end{aligned}
$$

\section{Proposed operational rules of single-valued complex neutrosophic sets}

The operational rules of two SVCNSs $H=\left(t_{H}(x) e^{j \pi \omega_{H}(x)}, i_{H} \cdot e^{j \pi \psi_{H}(x)}, f_{H}(x) \cdot e^{j \pi \phi_{H}(x)}\right)$ and $K=\left(t_{K}(x) e^{j \pi \omega_{K}(x)}, i_{K} \cdot e^{j \pi \psi_{K}(x)}, f_{K}(x) \cdot e^{j \pi \phi_{K}(x)}\right)$ are defined as the following:

(i) $H^{*} K=\left(T_{H * K}(x), I_{H * K}(x), F_{H * K}(x)\right)$

$$
=\left(\begin{array}{l}
t_{H}(x) t_{K}(x) \cdot e^{j \pi \omega_{\mathrm{H}}(x)^{*} \omega_{K}(x)},\left(i_{H}(x)+i_{K}(x)-i_{H}(x) i_{K}(x)\right) \cdot e^{j \pi \psi_{H}(x)^{*} \psi_{K}(x)}, \\
\left(f_{H}(x)+f_{K}(x)-f_{H}(x) f_{K}(x)\right) \cdot e^{j \pi \phi_{\mathrm{H}}(x)^{*} \phi_{K}(x)}
\end{array}\right)
$$

ii) $H+K=\left(T_{H+K}(x), I_{H+K}(x), F_{H+K}(x)\right)$ 


$$
=\left(\begin{array}{l}
{\left[t_{H}(x)+t_{K}(x)-t_{H}(x) t_{K}(x)\right] \cdot e^{j \omega\left[\omega_{H}(x)+\omega_{K}(x)\right]}, i_{H}(x) i_{K}(x) \cdot e^{j \pi\left[\psi_{H}(x)+\psi_{K}(x)\right]},} \\
f_{H}(x) f_{K}(x) \cdot e^{j \pi\left[\phi_{A}(x)+\phi_{B}(x)\right]}
\end{array}\right)
$$

(iii) $R=c . H=\left(T_{R}(x), I_{R}(x), F_{R}(x)\right)$

$$
=\left(\left[1-\left(1-t_{H}(x)\right)^{c}\right] \cdot e^{j \pi c^{*} \omega_{H}(x)},\left(i_{H}(x)\right)^{c} \cdot e^{j \pi c^{*} \psi_{H}(x)},\left(f_{H}(x)\right)^{c} \cdot e^{j \pi c^{*} \phi_{H}(x)}\right)
$$

\section{Proposed the new TOPSIS approach for distribution center location selection}

This section develops a new TOPSIS approach in SVCNS to select the most suitable distribution center. The steps of the proposed MCDM method are as follows:

Step 1: Determine and aggregate the ratings of distribution center locations versus criteria Step 2: Determine and aggregate the importance weights of criteria

Step 3: Calculate the weighted ratings of distribution center locations versus criteria Step 4: Rank the potential distribution center locations.

\subsection{Determining and aggregating the ratings of distribution center locations versus criteria}

Let $x_{u v k}=\left(T_{u v k}, I_{u v k}, F_{u v k}\right)$ be the suitability rating assigned to alternative $A_{u}, u=1, \ldots, m$ by $l$ decision-maker $D_{k}, k=1, \ldots, l$ for criterion $C_{v}, v=1, \ldots, n$, where: $T_{u v k}=t_{u v k} \cdot e^{j \pi \omega(x)}, \quad I_{u v k}=i_{u v k} \cdot e^{j \pi \psi(x)}$, $F_{u v k}=f_{u v k} \cdot e^{j \pi \phi(x)}$. Using the equations (1)-(3), the averaged suitability rating $x_{u v}=\left(T_{u v}, I_{u v}, F_{u v}\right)$ can be evaluated as:

$$
x_{u v}=\frac{1}{l} \otimes\left(x_{u v 1} \oplus x_{u v 2} \oplus \ldots \oplus x_{u v k} \oplus \ldots \oplus x_{u v l}\right),
$$

where,

$$
T_{u v}=\left[\wedge\left(\frac{1}{l} \sum_{k=1}^{l} t_{u v}, 1\right)\right] e^{j \pi\left[\frac{1}{l} \sum_{k=1}^{l} w_{k}(x)\right]} \quad I_{u v}=\left[\wedge\left(\frac{1}{l} \sum_{k=1}^{l} i_{u v}, 1\right)\right] e^{j \pi\left[\frac{1}{l} \sum_{k=1}^{l} \psi_{k}(x)\right]} \quad F_{u v}=\left[\wedge\left(\frac{1}{l} \sum_{k=1}^{l} f_{u v k}, 1\right)\right] e^{j \pi\left[\frac{1}{l} \sum_{k=1}^{l} \phi_{k}(x)\right]}
$$

\subsection{Determining and aggregating the importance weights of criteria}

Let $w_{u v}=\left(T_{u v}, I_{u v}, F_{u v}\right)$ be the weight assigned by decision-maker $D_{k}$ to criterion $C_{v}$, where: $T_{u v}=t_{u v} \cdot e^{j \pi \omega(x)}, I_{u v}=i_{u v} \cdot e^{j \pi \psi(x)}, F_{u v}=f_{u v} \cdot e^{j \pi \phi(x)}, F_{u v}=f_{u v} \cdot e^{j \pi \phi(x)}, v=1, \ldots, n ; k=1, \ldots, l$. Using the Eqs. (1)-(3), the aggregated weight $w_{v}=\left(T_{v}, I_{v}, F_{v}\right)$ can be evaluated as:

$$
w_{v}=\left(\frac{1}{l}\right) \otimes\left(w_{v 1} \oplus w_{v 2} \oplus \ldots \oplus w_{v l}\right)
$$

where,

$$
T_{v}=\left[\wedge\left(\frac{1}{l} \sum_{k=1}^{l} t_{v k}, 1\right)\right] e^{j \pi\left[\frac{1}{l} \sum_{k=1}^{l} w_{k}(x)\right]} \quad I_{v}=\left[\wedge\left(\frac{1}{l} \sum_{k=1}^{l} i_{v k}, 1\right)\right] e^{j \pi\left[\frac{1}{l} \sum_{k=1}^{l} \psi_{k}(x)\right]} \quad F_{v}=\left[\wedge\left(\frac{1}{l} \sum_{k=1}^{l} f_{v k}, 1\right)\right] e^{j \pi\left[\frac{1}{l} \sum_{k=1}^{l} \phi_{k}(x)\right]}
$$

\subsection{Calculating the weighted ratings of distribution center locations versus criteria}

Using the Eqs. (1)-(3), the weighted ratings of alternatives versus criteria can be calculated as: 


$$
G_{u}=\frac{1}{k} \sum_{v=1}^{l} x_{u v}^{*} w_{v}, u=1, \ldots, m ; v=1, \ldots, l
$$

\subsection{Ranking the potential distribution center locations}

This section developed the score, accuracy and certainty function for a SVCNS, i.e. $G_{u}=\left(T_{u}, I_{u}, F_{u}\right)$, where: $T_{u}=t_{u} \cdot e^{j \pi \omega(x)}, I_{u}=i_{u} \cdot e^{j \pi \psi(x)}, F_{u}=f_{u} \cdot e^{j \pi \phi(x)}, u=1, \ldots, m$, adopted from Peng et al. (2014) as follows:

The values of the score, accuracy and certainty function for amplitude terms are defined as follows:

$$
e_{G_{u}}^{a}=\left(2+t_{u}-i_{u}-f_{u}\right) / 3, h_{u}=t_{u}-f_{u} \text {, and } c_{u}=t_{u}
$$

The values of the score, accuracy and certainty function for phase terms are defined below:

$$
e_{G_{u}}^{p}=\pi[\omega(x)-\psi(x)-\phi(x)], h_{G_{u}}^{p}=\pi[\omega(x)-\phi(x)] \text {, and } c_{G_{u}}^{p}=\pi \omega(x)
$$

Let $G_{1}$ and $G_{2}$ be any two SVCNSs. Then, the ranking method can be defined as follows:

- If $e_{G_{1}}^{a}>e_{G_{2}}^{a}$, then $G_{1}>G_{2}$

- If $e_{G_{1}}^{a}=e_{G_{2}}^{a}$ and $e_{G_{1}}^{p}>e_{G_{2}}^{p}$, then $G_{1}>G_{2}$

- If $e_{G_{1}}^{a}=e_{G_{2}}^{a}, e_{G_{1}}^{p}=e_{G_{2}}^{p}$ and $h_{G_{1}}^{a}>h_{G_{2}}^{a}$, then $G_{1}>G_{2}$

- If $e_{G_{1}}^{a}=e_{G_{2}}^{a}, e_{G_{1}}^{p}=e_{G_{2}}^{p}, h_{G_{1}}^{a}=h_{G_{2}}^{a}$ and $h_{G_{1}}^{p}>h_{G_{2}}^{p}$, then $G_{1}>G_{2}$

- If $e_{G_{1}}^{a}=e_{G_{2}}^{a}, e_{G_{1}}^{p}=e_{G_{2}}^{p}, h_{G_{1}}^{a}=h_{G_{2}}^{a}, h_{G_{1}}^{p}=h_{G_{2}}^{p}$ and $c_{G_{1}}^{a}>c_{G_{2}}^{a}$, then $G_{1}>G_{2}$

- If $e_{G_{1}}^{a}=e_{G_{2}}^{a}, e_{G_{1}}^{p}=e_{G_{2}}^{p}, h_{G_{1}}^{a}=h_{G_{2}}^{a}, h_{G_{1}}^{p}=h_{G_{2}}^{p}, c_{G_{1}}^{a}=c_{G_{2}}^{a}$ and $c_{G_{1}}^{p}>c_{G_{2}}^{p}$, then $G_{1}>G_{2}$

- If $e_{G_{1}}^{a}=e_{G_{2}}^{a}, e_{G_{1}}^{p}=e_{G_{2}}^{p}, h_{G_{1}}^{a}=h_{G_{2}}^{a}, h_{G_{1}}^{p}=h_{G_{2}}^{p}, c_{G_{1}}^{a}=c_{G_{2}}^{a}$ and $c_{G_{1}}^{p}=c_{G_{2}}^{p}$, then $G_{1}=G_{2}$

\section{Application of the proposed TOPSIS approach for distribution center location selection}

In this section, the proposed TOPSIS approach in SVCNS is applied for distribution center location selection in the case of an industrial company. Assume that the company needs to select a location to establish a new distribution center for expanding their business. After preliminary screening, four locations, namely $A_{1}, A_{2}, A_{3}$ and $A_{4}$, are chosen for further selection. A committee of three company managers, i.e. $D_{1}, D_{2}$, and $D_{3}$, were required to make their evaluation separately, according to their preferences for the importance weights of criteria and the ratings of alternative based on each criterion. Six criteria were chosen from the literature in order to select the distribution center location including transportation availability $\left(C_{1}\right)$, closeness to demand market $\left(C_{2}\right)$, human resources $\left(C_{3}\right)$, availability of acquirement material $\left(C_{4}\right)$, investment cost $\left(C_{5}\right)$, and expansion possibility $\left(C_{6}\right)$. The computational procedure is summarized as follows:

\subsection{Determining and Aggregating the Ratings of Locations versus the Criteria}

The committee members use the linguistic rating set $S=\{V L, L, F, G, V G\}$, where VL $=$ Very Low $=$ $\left(0.1 . \mathrm{e}^{\mathrm{j} \pi 0.7}, 0.7 . \mathrm{e}^{\mathrm{j} \pi 0.9}, 0.6 . \mathrm{e}^{\mathrm{j} \pi 1.0}\right), \mathrm{L}=$ Low $=\left(0.3 . \mathrm{e}^{\mathrm{j} \pi 0.8}, 0.6 . \mathrm{e}^{\mathrm{j} \pi 1.0}, 0.5 \cdot \mathrm{e}^{\mathrm{j} \pi 0.9}\right), \mathrm{F}=$ Fair $=\left(0.4 . \mathrm{e}^{\mathrm{j} \pi 0.8}\right.$, 0.5. $\left.\mathrm{e}^{\mathrm{j} \pi 0.9}, 0.4 . \mathrm{e}^{\mathrm{j} \pi 0.8}\right), \mathrm{G}=$ Good $=\left(0.6 . \mathrm{e}^{\mathrm{j} \pi 0.9}, 0.4 . \mathrm{e}^{\mathrm{j} \pi 0.9}, 0.3 . \mathrm{e}^{\mathrm{j} \pi 0.7}\right)$, and VG $=$ Very Good $=\left(0.7 . \mathrm{e}^{\mathrm{j} \pi}\right.$ $\left.1.1,0.2 . \mathrm{e}^{\mathrm{j} \pi 0.8}, 0.1 \mathrm{e}^{\mathrm{j} \pi 0.6}\right)$, to evaluate the suitability of the locations under each criteria. Table 1 gives the aggregated ratings of four locations $\left(A_{1}, A_{2}, A_{3}, A_{4}\right)$ versus six criteria $\left(C_{1}, . ., C_{6}\right)$ from three decisionmakers $\left(D_{1}, D_{2}, D_{3}\right)$ using Eq. (4). 
Table 1

Aggregated ratings of locations versus the criteria

\begin{tabular}{|c|c|c|c|c|c|}
\hline \multirow{2}{*}{ Criteria } & \multirow{2}{*}{ Locations } & \multicolumn{3}{|c|}{ Decision-makers } & \multirow{2}{*}{ Aggregated ratings } \\
\hline & & $D_{1}$ & $D_{2}$ & $D_{3}$ & \\
\hline \multirow{4}{*}{$C_{1}$} & $A_{1}$ & M & G & G & $\left(0.353 . \mathrm{e}^{\mathrm{j} \pi 0.65}, 0.622 . \mathrm{e}^{\mathrm{j} \pi 0.575}, 0.532 . \mathrm{e}^{\mathrm{j} \pi 0.5}\right)$ \\
\hline & $A_{2}$ & G & G & M & $\left(0.353 . \mathrm{e}^{\mathrm{j} \pi 0.65}, 0.622 . \mathrm{e}^{\mathrm{j} \pi 0.575}, 0.532 . \mathrm{e}^{\mathrm{j} \pi 0.5}\right)$ \\
\hline & $A_{3}$ & VG & G & G & $\left(0.438 . \mathrm{e}^{\mathrm{j} \pi 0.7}, 0.523 . \mathrm{e}^{\mathrm{j} \pi 0.625}, 0.468 . \mathrm{e}^{\mathrm{j} \pi 0.55}\right)$ \\
\hline & $A_{4}$ & G & M & G & $\left(0.353 . \mathrm{e}^{\mathrm{j} \pi 0.65}, 0.622 . \mathrm{e}^{\mathrm{j} \pi 0.575}, 0.532 . \mathrm{e}^{\mathrm{j} \pi 0.5}\right)$ \\
\hline \multirow{4}{*}{$C_{2}$} & $A_{1}$ & G & G & G & $\left(0.6 . \mathrm{e}^{\mathrm{j} \pi 0.9}, 0.4 . \mathrm{e}^{\mathrm{j} \pi 0.9}, 0.3 . \mathrm{e}^{\mathrm{j} \pi 0.7}\right)$ \\
\hline & $A_{2}$ & G & G & G & $\left(0.6 . \mathrm{e}^{\mathrm{j} \pi 0.9}, 0.4 . \mathrm{e}^{\mathrm{j} \pi 0.9}, 0.3 . \mathrm{e}^{\mathrm{j} \pi 0.7}\right)$ \\
\hline & $A_{3}$ & M & G & G & $\left(0.353 . \mathrm{e}^{\mathrm{j} \pi 0.65}, 0.622 . \mathrm{e}^{\mathrm{j} \pi 0.575}, 0.532 . \mathrm{e}^{\mathrm{j} \pi 0.5}\right)$ \\
\hline & $A_{4}$ & VG & $\mathrm{G}$ & $\mathrm{G}$ & $\left(0.438 . \mathrm{e}^{\mathrm{j} \pi 0.7}, 0.523 . \mathrm{e}^{\mathrm{j} \pi 0.625}, 0.468 . \mathrm{e}^{\mathrm{j} \pi 0.55}\right)$ \\
\hline \multirow{4}{*}{$C_{3}$} & $A_{1}$ & $\mathrm{M}$ & G & G & $\left(0.353 . \mathrm{e}^{\mathrm{j} \pi 0.65}, 0.622 . \mathrm{e}^{\mathrm{j} \pi 0.575}, 0.532 . \mathrm{e}^{\mathrm{j} \pi 0.5}\right)$ \\
\hline & $A_{2}$ & G & G & G & $\left(0.6 . \mathrm{e}^{\mathrm{j} \pi 0.9}, 0.4 . \mathrm{e}^{\mathrm{j} \pi 0.9}, 0.3 . \mathrm{e}^{\mathrm{j} \pi 0.7}\right)$ \\
\hline & $A_{3}$ & VG & G & VG & $\left(0.468 . \mathrm{e}^{\mathrm{j} \pi 0.725}, 0.461 . \mathrm{e}^{\mathrm{j} \pi 0.65}, 0.436 . \mathrm{e}^{\mathrm{j} \pi 0.575}\right)$ \\
\hline & $A_{4}$ & G & VG & $\mathrm{G}$ & $\left(0.438 . \mathrm{e}^{\mathrm{j} \pi 0.7}, 0.523 . \mathrm{e}^{\mathrm{j} \pi 0.625}, 0.468 . \mathrm{e}^{\mathrm{j} \pi 0.55}\right)$ \\
\hline \multirow{4}{*}{$C_{4}$} & $A_{1}$ & M & $\mathrm{P}$ & M & $\left(0.209 . \mathrm{e}^{\mathrm{j} \pi 0.575}, 0.682 . \mathrm{e}^{\mathrm{j} \pi 0.5}, 0.647 . \mathrm{e}^{\mathrm{j} \pi 0.425}\right)$ \\
\hline & $A_{2}$ & M & G & G & $\left(0.353 . \mathrm{e}^{\mathrm{j} \pi 0.65}, 0.622 . \mathrm{e}^{\mathrm{j} \pi 0.575}, 0.532 . \mathrm{e}^{\mathrm{j} \pi 0.5}\right)$ \\
\hline & $A_{3}$ & M & M & G & $\left(0.296 . \mathrm{e}^{\mathrm{j} \pi 0.625}, 0.651 . \mathrm{e}^{\mathrm{j} \pi 0.55}, 0.562 . \mathrm{e}^{\mathrm{j} \pi 0.475}\right)$ \\
\hline & $A_{4}$ & G & $\mathrm{G}$ & $\mathrm{G}$ & $\left(0.6 . \mathrm{e}^{\mathrm{j} \pi 0.9}, 0.4 . \mathrm{e}^{\mathrm{j} \pi 0.9}, 0.3 . \mathrm{e}^{\mathrm{j} \pi 0.7}\right)$ \\
\hline \multirow{4}{*}{$C_{5}$} & $A_{1}$ & G & $\mathrm{M}$ & G & $\left(0.353 . \mathrm{e}^{\mathrm{j} \pi 0.65}, 0.622 . \mathrm{e}^{\mathrm{j} \pi 0.575}, 0.532 . \mathrm{e}^{\mathrm{j} \pi 0.5}\right)$ \\
\hline & $A_{2}$ & G & G & G & $\left(0.6 . \mathrm{e}^{\mathrm{j} \pi 0.9}, 0.4 . \mathrm{e}^{\mathrm{j} \pi 0.9}, 0.3 . \mathrm{e}^{\mathrm{j} \pi 0.7}\right)$ \\
\hline & $A_{3}$ & G & G & VG & $\left(0.438 . \mathrm{e}^{\mathrm{j} \pi 0.7}, 0.523 . \mathrm{e}^{\mathrm{j} \pi 0.625}, 0.468 . \mathrm{e}^{\mathrm{j} \pi 0.55}\right)$ \\
\hline & $A_{4}$ & G & G & $\mathrm{G}$ & $\left(0.6 . \mathrm{e}^{\mathrm{j} \pi 0.9}, 0.4 . \mathrm{e}^{\mathrm{j} \pi 0.9}, 0.3 . \mathrm{e}^{\mathrm{j} \pi 0.7}\right)$ \\
\hline \multirow{4}{*}{$C_{6}$} & $A_{1}$ & VG & G & G & $\left(0.438 . \mathrm{e}^{\mathrm{j} \pi 0.7}, 0.523 . \mathrm{e}^{\mathrm{j} \pi 0.625}, 0.468 . \mathrm{e}^{\mathrm{j} \pi 0.55}\right)$ \\
\hline & $A_{2}$ & G & G & G & $\left(0.6 . \mathrm{e}^{\mathrm{j} \pi 0.9}, 0.4 . \mathrm{e}^{\mathrm{j} \pi 0.9}, 0.3 . \mathrm{e}^{\mathrm{j} \pi 0.7}\right)$ \\
\hline & $A_{3}$ & VG & G & VG & $\left(0.468 . \mathrm{e}^{\mathrm{j} \pi 0.725}, 0.461 . \mathrm{e}^{\mathrm{j} \pi 0.65}, 0.436 . \mathrm{e}^{\mathrm{j} \pi 0.575}\right)$ \\
\hline & $A_{4}$ & $\mathrm{G}$ & M & $\mathrm{G}$ & $\left(0.353 . \mathrm{e}^{\mathrm{j} \pi 0.65}, 0.622 . \mathrm{e}^{\mathrm{j} \pi 0.575}, 0.532 . \mathrm{e}^{\mathrm{j} \pi 0.5}\right)$ \\
\hline
\end{tabular}

\subsection{Determining and aggregating the importance weights of criteria}

The committee members' opinions on the importance of criteria are expressed using a linguistic weighting set $\mathrm{K}=\{\mathrm{UI}, \mathrm{OI}, \mathrm{I}, \mathrm{VI}, \mathrm{AI}\}$ where $\mathrm{UI}=$ Unimportant $=\left(0.2 . \mathrm{e}^{\mathrm{j} \pi 0.7}, 0.5 . \mathrm{e}^{\mathrm{j} \pi 0.9}, 0.5 \mathrm{e}^{\mathrm{j} \pi 1.1}\right)$, OI $=$ Ordinary Important $=\left(0.3 . \mathrm{e}^{\mathrm{j} \pi 0.8}, 0.5 . \mathrm{e}^{\mathrm{j} \pi 1.0}, 0.4 . \mathrm{e}^{\mathrm{j} \pi 0.9}\right), \mathrm{I}=$ Important $=\left(0.5 \cdot \mathrm{e}^{\mathrm{j} \pi 0.9}, 0.4 . \mathrm{e}^{\mathrm{j} \pi 0.9}, 0.3 \cdot \mathrm{e}^{\mathrm{j}}\right.$ $\pi 0.8)$, VI $=$ Very Important $=\left(0.7 . \mathrm{e}^{\mathrm{j} \pi 0.9}, 0.3 . \mathrm{e}^{\mathrm{j} \pi 0.9}, 0.2 \cdot \mathrm{e}^{\mathrm{j} \pi 0.7}\right)$, and AI $=$ Absolutely Important $=\left(0.8 . \mathrm{e}^{\mathrm{j}}\right.$ $\pi 1.0,\left[0.2 . \mathrm{e}^{\mathrm{j} \pi 0.8}, 0.1 . \mathrm{e}^{\mathrm{j} \pi 0.6}\right)$. Table 2 displays the importance weights of the six criteria from the three decision-makers. The aggregated weights of criteria obtained by Eq. (5) are shown in the last column of Table 2.

Table 2

The importance and aggregated weights of the criteria

\begin{tabular}{|c|c|c|c|c|}
\hline \multirow{2}{*}{ Criteria } & \multicolumn{3}{|c|}{ Decision-makers } & \multirow{2}{*}{ Aggregated weights } \\
\hline & $D_{1}$ & $D_{2}$ & $D_{3}$ & \\
\hline$C_{1}$ & VI & I & I & $\left(0.477 . \mathrm{e}^{\mathrm{j} \pi 0.675}, 0.468 . \mathrm{e}^{\mathrm{j} \pi 0.675}, 0.366 . \mathrm{e}^{\mathrm{j} \pi 0.575}\right)$ \\
\hline$C_{2}$ & I & I & OI & $\left(0.353 . \mathrm{e}^{\mathrm{j} \pi 0.650}, 0.532 . \mathrm{e}^{\mathrm{j} \pi 0.7}, 0.436 . \mathrm{e}^{\mathrm{j} \pi 0.625}\right)$ \\
\hline$C_{3}$ & I & VI & I & $\left(0.457 . \mathrm{e}^{\mathrm{j} \pi 0.675}, 0.468 . \mathrm{e}^{\mathrm{j} \pi 0.675}, 0.366 . \mathrm{e}^{\mathrm{j} \pi 0.575}\right)$ \\
\hline$C_{4}$ & AI & VI & AI & $\left(0.669 . \mathrm{e}^{\mathrm{j} \pi 0.725}, 0.331 . \mathrm{e}^{\mathrm{j} \pi 0.625}, 0.211 . \mathrm{e}^{\mathrm{j} \pi 0.475}\right)$ \\
\hline$C_{5}$ & VI & VI & $\mathrm{AI}$ & $\left(0.634 . \mathrm{e}^{\mathrm{j} \pi 0.7}, 0.366 . \mathrm{e}^{\mathrm{j} \pi 0.65}, 0.251 . \mathrm{e}^{\mathrm{j} \pi 0.5}\right)$ \\
\hline$C_{6}$ & I & I & VI & $\left(0.457 . \mathrm{e}^{\mathrm{j} \pi 0.675}, 0.468 . \mathrm{e}^{\mathrm{j} \pi 0.675}, 0.366 . \mathrm{e}^{\mathrm{j} \pi 0.575}\right)$ \\
\hline
\end{tabular}




\subsection{Compute the aggregate weighted rating of each location}

The aggregate weighted rating value of each location can be obtained by multiplying the aggregated ratings of locations and the aggregated weight of criteria using Eq. (6) (as shown in Table 3). Table 4 presents the final average evaluation values of each location.

\section{Table 3}

Aggregate weighted rating value of each location

Criteria Locations

Aggregate weighted ratings

\begin{tabular}{|c|c|c|}
\hline \multirow{4}{*}{$C_{1}$} & $A_{1}$ & $\left(0.125 . \mathrm{e}^{\mathrm{j} \pi 0.423}, 0.823 . \mathrm{e}^{\mathrm{j} \pi 0.403}, 0.781 . \mathrm{e}^{\mathrm{j} \pi 0.35}\right)$ \\
\hline & $A_{2}$ & $\left(0.125 . \mathrm{e}^{\mathrm{j} \pi 0.423}, 0.823 . \mathrm{e}^{\mathrm{j} \pi 0.403}, 0.781 . \mathrm{e}^{\mathrm{j} \pi 0.35}\right)$ \\
\hline & $A_{3}$ & $\left(0.155 . \mathrm{e}^{\mathrm{j} \pi 0.455}, 0.777 . \mathrm{e}^{\mathrm{j} \pi 0.438}, 0.751 . \mathrm{e}^{\mathrm{j} \pi 0.385}\right)$ \\
\hline & $A_{4}$ & $\left(0.125 . \mathrm{e}^{\mathrm{j} \pi 0.423}, 0.823 . \mathrm{e}^{\mathrm{j} \pi 0.403}, 0.781 . \mathrm{e}^{\mathrm{j} \pi 0.35}\right)$ \\
\hline \multirow{4}{*}{$C_{2}$} & $A_{1}$ & $\left(0.143 . \mathrm{e}^{\mathrm{j} \pi 0.439}, 0.81 . \mathrm{e}^{\mathrm{j} \pi 0.42}, 0.767 . \mathrm{e}^{\mathrm{j} \pi 0.368}\right)$ \\
\hline & $A_{2}$ & $\left(0.143 . \mathrm{e}^{\mathrm{j} \pi 0.439}, 0.81 . \mathrm{e}^{\mathrm{j} \pi 0.42}, 0.767 . \mathrm{e}^{\mathrm{j} \pi 0.368}\right)$ \\
\hline & $A_{3}$ & $\left(0.125 . \mathrm{e}^{\mathrm{j} \pi 0.423}, 0.823 . \mathrm{e}^{\mathrm{j} \pi 0.403}, 0.781 . \mathrm{e}^{\mathrm{j} \pi 0.35}\right)$ \\
\hline & $A_{4}$ & $\left(0.155 . \mathrm{e}^{\mathrm{j} \pi 0.455}, 0.777 . \mathrm{e}^{\mathrm{j} \pi 0.438}, 0.751 . \mathrm{e}^{\mathrm{j} \pi 0.385}\right)$ \\
\hline \multirow{4}{*}{$C_{3}$} & $A_{1}$ & $\left(0.143 . \mathrm{e}^{\mathrm{j} \pi 0.439}, 0.81 . \mathrm{e}^{\mathrm{j} \pi 0.42}, 0.767 . \mathrm{e}^{\mathrm{j} \pi 0.368}\right)$ \\
\hline & $A_{2}$ & $\left(0.164 . \mathrm{e}^{\mathrm{j} \pi 0.456}, 0.799 . \mathrm{e}^{\mathrm{j} \pi 0.405}, 0.753 . \mathrm{e}^{\mathrm{j} \pi 0.354}\right)$ \\
\hline & $A_{3}$ & $\left(0.19 . \mathrm{e}^{\mathrm{j} \pi 0.489}, 0.732 . \mathrm{e}^{\mathrm{j} \pi 0.439}, 0.719 . \mathrm{e}^{\mathrm{j} \pi 0.388}\right)$ \\
\hline & $A_{4}$ & $\left(0.177 . \mathrm{e}^{\mathrm{j} \pi 0.473}, 0.763 . \mathrm{e}^{\mathrm{j} \pi 0.422}, 0.736 . \mathrm{e}^{\mathrm{j} \pi 0.371}\right)$ \\
\hline \multirow{4}{*}{$C_{4}$} & $A_{1}$ & $\left(0.14 . \mathrm{e}^{\mathrm{j} \pi 0.417}, 0.787 . \mathrm{e}^{\mathrm{j} \pi 0.313}, 0.764 . \mathrm{e}^{\mathrm{j} \pi 0.266}\right)$ \\
\hline & $A_{2}$ & $\left(0.236 . \mathrm{e}^{\mathrm{j} \pi 0.471}, 0.747 . \mathrm{e}^{\mathrm{j} \pi 0.359}, 0.687 . \mathrm{e}^{\mathrm{j} \pi 0.313}\right)$ \\
\hline & $A_{3}$ & $\left(0.198 . \mathrm{e}^{\mathrm{j} \pi 0.453}, 0.767 . \mathrm{e}^{\mathrm{j} \pi 0.344}, 0.707 . \mathrm{e}^{\mathrm{j} \pi 0.297}\right)$ \\
\hline & $A_{4}$ & $\left(0.271 . \mathrm{e}^{\mathrm{j} \pi 0.489}, 0.729 . \mathrm{e}^{\mathrm{j} \pi 0.375}, 0.667 . \mathrm{e}^{\mathrm{j} \pi 0.328}\right)$ \\
\hline \multirow{4}{*}{$C_{5}$} & $A_{1}$ & $\left(0.191 . \mathrm{e}^{\mathrm{j} \pi 0.439}, 0.787 . \mathrm{e}^{\mathrm{j} \pi 0.388}, 0.736 . \mathrm{e}^{\mathrm{j} \pi 0.338}\right)$ \\
\hline & $A_{2}$ & $\left(0.219 . \mathrm{e}^{\mathrm{j} \pi 0.456}, 0.771 . \mathrm{e}^{\mathrm{j} \pi 0.405}, 0.719 . \mathrm{e}^{\mathrm{j} \pi 0.354}\right)$ \\
\hline & $A_{3}$ & $\left(0.236 . \mathrm{e}^{\mathrm{j} \pi 0.473}, 0.731 . \mathrm{e}^{\mathrm{j} \pi 0.422}, 0.7 . \mathrm{e}^{\mathrm{j} \pi 0.371}\right)$ \\
\hline & $A_{4}$ & $\left(0.219 . \mathrm{e}^{\mathrm{j} \pi 0.456}, 0.771 . \mathrm{e}^{\mathrm{j} \pi 0.405}, 0.719 . \mathrm{e}^{\mathrm{j} \pi 0.354}\right)$ \\
\hline \multirow{4}{*}{$C_{6}$} & $A_{1}$ & $\left(0.236 . \mathrm{e}^{\mathrm{j} \pi 0.473}, 0.731 . \mathrm{e}^{\mathrm{j} \pi 0.422}, 0.7 . \mathrm{e}^{\mathrm{j} \pi 0.371}\right)$ \\
\hline & $A_{2}$ & $\left(0.219 . \mathrm{e}^{\mathrm{j} \pi 0.456}, 0.771 . \mathrm{e}^{\mathrm{j} \pi 0.405}, 0.719 . \mathrm{e}^{\mathrm{j} \pi 0.354}\right)$ \\
\hline & $A_{3}$ & $\left(0.253 . \mathrm{e}^{\mathrm{j} \pi 0.489}, 0.696 . \mathrm{e}^{\mathrm{j} \pi 0.439}, 0.681 . \mathrm{e}^{\mathrm{j} \pi 0.388}\right)$ \\
\hline & $A_{4}$ & $\left(0.191 . \mathrm{e}^{\mathrm{j} \pi 0.439}, 0.787 . \mathrm{e}^{\mathrm{j} \pi 0.388}, 0.736 . \mathrm{e}^{\mathrm{j} \pi 0.338}\right)$ \\
\hline
\end{tabular}

Table 4

The final evaluation values of each location

Locations

Aggregated weights

\begin{tabular}{ll}
\hline$A_{1}$ & $\left(0.164 . \mathrm{e}^{\mathrm{j} \pi 0.438}, 0.791 . \mathrm{e}^{\mathrm{j} \pi 0.389}, 0.752 . \mathrm{e}^{\mathrm{j} \pi 0.338}\right)$ \\
$A_{2}$ & $\left(0.185 . \mathrm{e}^{\mathrm{j} \pi 0.45}, 0.787 . \mathrm{e}^{\mathrm{j} \pi 0.399}, 0.737 . \mathrm{e}^{\mathrm{j} \pi 0.349}\right)$ \\
$A_{3}$ & $\left(0.194 . \mathrm{e}^{\mathrm{j} \pi 0.464}, 0.753 . \mathrm{e}^{\mathrm{j} \pi 0.414}, 0.723 . \mathrm{e}^{\mathrm{j} \pi 0.363}\right)$ \\
$A_{4}$ & $\left(0.191 . \mathrm{e}^{\mathrm{j} \pi 0.456}, 0.774 . \mathrm{e}^{\mathrm{j} \pi 0.405}, 0.731 . \mathrm{e}^{\mathrm{j} \pi 0.354}\right)$ \\
\hline
\end{tabular}

\subsection{Ranking the alternatives}

Using the proposed the score, accuracy and certainty function for a SVCNS in Equations (7)-(8), the final ranking value of each location is calculated as in Table 5. According to this table, the ranking order of the four locations is $A_{3} \succ A_{4} \succ A_{2} \succ A_{1}$. 
Table 5

Modified score function of each alternative

\begin{tabular}{cccccccc}
\hline \multirow{2}{*}{ Locations } & \multicolumn{2}{c}{$\begin{array}{c}\text { Proposed score } \\
\text { function }\end{array}$} & \multicolumn{2}{c}{$\begin{array}{c}\text { Proposed accuracy } \\
\text { function }\end{array}$} & \multicolumn{2}{c}{$\begin{array}{c}\text { Proposed certainty } \\
\text { function }\end{array}$} & Ranking \\
\cline { 2 - 7 } & $\begin{array}{c}\text { Amplitude } \\
\text { term }\end{array}$ & $\begin{array}{c}\text { Phase } \\
\text { term }\end{array}$ & $\begin{array}{c}\text { Amplitude } \\
\text { term }\end{array}$ & $\begin{array}{c}\text { Phase } \\
\text { term }\end{array}$ & $\begin{array}{c}\text { Amplitude } \\
\text { term }\end{array}$ & $\begin{array}{c}\text { Phase } \\
\text { term }\end{array}$ & \\
\hline$A_{1}$ & 0,207 & $-0,289 \pi$ & $-0,588$ & $0,100 \pi$ & 0,164 & $0,438 \pi$ & $\mathbf{4}$ \\
$A_{2}$ & 0,221 & $-0,298 \pi$ & $-0,552$ & $0,101 \pi$ & 0,185 & $0,450 \pi$ & $\mathbf{3}$ \\
$A_{3}$ & 0,239 & $-0,313 \pi$ & $-0,529$ & $0,100 \pi$ & 0,194 & $0,464 \pi$ & $\mathbf{1}$ \\
$A_{4}$ & 0,229 & $-0,304 \pi$ & $-0,540$ & $0,101 \pi$ & 0,191 & $0,456 \pi$ & $\mathbf{2}$ \\
\hline
\end{tabular}

\section{Conclusion}

This paper has proposed new TOPSIS approach in complex neutrosophic set for solving distribution location selection and evaluation. The operational rules of the SVCNS were also defined. In the proposed TOPSIS approach, the importance weights of decision criteria and the ratings of qualitative criteria were assessed in linguistic variables which are described by complex neutrosophic sets. The proposed approach was then applied to solve the distribution location selection and evaluation problem with three decision makers and six selection criteria. It has been demonstrated throughout the detailed calculation in the application that the proposed decision-making methods are efficient and more general as compared to the relevant studies. The proposed approach can also be applied to other management problems under similar settings.

\section{References}

Abdel-Basset, M., \& Mohamed, M. (2019). A novel and powerful framework based on neutrosophic sets to aid patients with cancer. Future Generation Computer Systems, 98, 144-153.

Abdel-Basset, M., Chang, V., Gamal, A., \& Smarandache, F. (2019). An integrated neutrosophic ANP and VIKOR method for achieving sustainable supplier selection: A case study in importing field. Computers in Industry, 106, 94-110.

Ali, M., \& Smarandache, F. (2017). Complex neutrosophic set. Neural Computing and Applications, 28(7), 1817-1834.

Ali, M., Dat, L.Q., Son, L.H., \& Smarandache, F. (2018). Interval Complex Neutrosophic Set: Formulation and Applications in Decision-Making. International Journal of Fuzzy Systems, 20(3), 986-999.

Awasthi, A., Chauhan, S. S., \& Goyal, S. K. (2011). A multi-criteria decision-making approach for location planning for urban distribution centers under uncertainty. Mathematical \& Computer Modelling, 53(1-2), 98-09.

Chu, T. C., \& Hsu, W. C. (2016). Evaluating Distribution Centers via a Maximizing Set and Minimizing Set Based Fuzzy MCDM Approach. Journal of Business and Economics, 7(1), 81-93.

Chen, C. T. (2001). A fuzzy approach to select the location of the distribution center. Fuzzy Sets and Systems, 118(1), 65-73.

Tamir, D. E., Rishe, N. D., \& Kandel, A. (2015). Complex Fuzzy Sets and Complex Fuzzy Logic an Overview of Theory and Applications in D.E. Tamir et al. (eds.), Fifty Years of Fuzzy Logic and its Applications, Studies in Fuzziness and Soft Computing, 326, 661-681.

Das, S., Kumar, S., Kar, S., \& Pal, T. (2019). Group decision making using neutrosophic soft matrix: An algorithmic approach. Journal of King Saud University-Computer and Information Sciences, 31(4), 459-468.

Greenfield, S., Chiclana, F., \& Dick, S. (2016, July). Interval-valued complex fuzzy logic. In 2016 IEEE International Conference on Fuzzy Systems (FUZZ-IEEE) (pp. 2014-2019). IEEE.

Hua, X., Hu, X., \& Yuan, W. (2016). Research optimization on logistics distribution center location based on adaptive particle swarm algorithm. Optik - International Journal for Light and Electron Optics, 127(20), 8443-8450. 
Hiassat, A., Diabat, A., \& Rahwan, I. (2017). A genetic algorithm approach for location-inventoryrouting problem with perishable products. Journal of Manufacturing Systems, 42, 93-103.

Li, S., \& Wei, Z. (2018) A hybrid approach based on the analytic hierarchy process and 2-tuple hybrid ordered weighted averaging for location selection of distribution centers, PLoS ONE, 13(11), e0206966.

Liu, P., \& You, X. (2019). Bidirectional projection measure of linguistic neutrosophic numbers and their application to multi-criteria group decision making. Computers \& Industrial Engineering, 128 , 447-457.

Peng, J. J., Wang, J. Q., Wang, J., Zhang, H. Y., \& Chen, X. H. (2014). Simplified neutrosophic sets and their applications in multi-criteria group decision-making problems. International Journal of Systems Science, 47(10), 2342-2358.

Pham, T. Y., Ma, H. M., Yeo, G. T. (2017). Application of fuzzy delphi TOPSIS to locate logistics centers in Vietnam: The Logisticians' perspective. Asian Journal of Shipping \& Logistics, 33(4), 211-219.

Pramanik, S., Dalapati, S., \& Roy, T. K. (2016). Logistics Center Location Selection Approach Based on Neutrosophic Multi-Criteria Decision Making, 161-174; in Florentin Smarandache, Surapati Pramanik. New Trends in Neutrosophic Theory and Applications. 2016. hal-01408066.

Rahmani, A., \& Mirhassani, S. A. (2014). A hybrid Firefly-Genetic Algorithm for the capacitated facility location problem. Information Sciences, 283(4), 70-78.

Rao, C., Goh, M., Zhao, Y., \& Zheng, J. (2015). Location selection of city logistics centers under sustainability. Transportation Research Part D Transport \& Environment, 36, 29-44.

Ramot, D., Milo, R., Friedman, M., \& Kandel, A. (2002). Complex fuzzy sets. IEEE Transactions on Fuzzy Systems, 10(2), 171-186.

Smarandache, F. (1998). Neutrosophy: Neutrosophic Probability, Set, and Logic. Analytic Synthesis Synthetic Analysis. American Research Press.

Selvachandran, G., Pal, M., Alhawari, T. A. A., \& Salleh, A. R. (2018). Interval-valued complex fuzzy sets and its application to the Malaysian economy. International Journal of Fuzzy System Applications, 7(1), 22-31.

Selvachandran, G., Pal, M., Alhawari, T. A. A., \& Salleh, A. R. (2018). Interval-Valued Complex Fuzzy Sets and Its Application to the Malaysian Economy. International Journal of Fuzzy System Applications, 7(1), 22-31.

Singh, R. K., Chaudhary, N., \& Saxena, N. (2018). Selection of warehouse location for a global supply chain: A case study. IIMB Management Review, 30, 343-356.

Sodenkamp, M. A., Tavana, M., \& Caprio, D. D. (2018). An aggregation method for solving group multi-criteria decision-making problems with single-valued neutrosophic sets. Applied Soft Computing, 71, 715-727.

Toro, E. M., Franco, J. F., \& Echeverri, M. G. (2017). A Multi-Objective Model for the Green Capacitated Location-Routing Problem Considering Environmental Impact. Computers \& Industrial Engineering, 110, 114-125.

Wang, H., Smarandache, F., Zhang, Y., \& Sunderraman, R. (2005). Single valued neutrosophic sets, Proc Of 10th 476 Int Conf on Fuzzy Theory and Technology, Salt Lake City, 477 Utah. 2005, from: http://fs.unm.edu/SingleValuedNeutrosophicSets.pdf

Wang, H., Smarandache, F., Sunderraman, R., \& Zhang, Y. Q. (2005). Interval Neutrosophic Sets and Logic: Theory and Applications in Computing. Logic in Computer Science. arXiv:cs/0505014 [cs.LO].

Yazdanbakhsh, O., \& Dick, C. (2018). A systematic review of complex fuzzy sets and logic. Fuzzy Sets and Systems, 338(1), 1-22.

Zhalechian, M., Tavakkoli-Moghaddam R., \& Rahimi, Y. (2017). A self-adaptive evolutionary algorithm for a fuzzy multi-objective hub location problem: An integration of responsiveness and social responsibility. Engineering Applications of Artificial Intelligence, 62, 1-16. 
C 2020 by the authors; licensee Growing Science, Canada. This is an open access article distributed under the terms and conditions of the Creative Commons Attribution (CC-BY) license (http://creativecommons.org/licenses/by/4.0/). 